Instituto Internacional de Investigación y Desarrollo Tecnológico Educativo INDTEC, C.A.

DOI: https://doi.org/10.29394/Scientific.issn.2542-2987.2020.5.17.7.143-163

OAI-PMH: http://www.indteca.com/ojs/index.php/Revista Scientific/oai

Artículo Original / Original Article

\title{
Actividades Lúdicas como Estrategias de Transición Educativa
}

\author{
Autora: Marlise del Valle Parra Peña \\ Universidad Pedagógica Experimental Libertador, UPEL \\ mdvpp4@gmail.com \\ Barinas, Venezuela \\ https://orcid.org/0000-0001-9690-4773
}

\section{Resumen}

La investigación tuvo como propósito implementar la actividad lúdica como estrategia de aprendizaje que facilite el proceso de transición en los niños y niñas de inicial a primer grado de primaria de la escuela Básica "María La Riva Salas", municipio Barinas, estado Barinas, Venezuela. Metodológicamente la indagación se enmarcó en el paradigma socio crítico mediante el método de investigación acción. Los informantes, quedaron constituidos por cuatro (4) docentes: dos (2) de educación inicial y dos (2) de primaria. Las técnicas de recolección de información quedaron determinadas por la entrevista en profundidad, con el registro de notas de campo. Para la interpretación de información se utilizó las técnicas de categorización, triangulación y la teorización. Se destaca como hallazgos de la investigación, la falta de actividades recreativas que le permitan al estudiante adaptarse a la situación de aprendizaje en primer grado. Dentro de las reflexiones finales, se tiene que existe la necesidad de introducir cambios a través de actividades lúdicas en la praxis del docente para articular no solo los aspectos cognitivos, corporales, motrices, sino también, las relaciones con aprendizaje, en los cuales el estudiante podrá sentirse sin temores, incertidumbres o ansiedades, facilitando su incorporación en la educación primaria.

Palabras clave: juego educativo; aprendizaje; adaptación del estudiante.

Cómo citar este artículo:

Parra, M. (2020). Actividades Lúdicas como Estrategias de Transición Educativa. Revista Scientific, 5(17), 143-163, e-ISSN: 2542-2987. Recuperado de: https://doi.org/10.29394/Scientific.issn.2542-2987.2020.5.17.7.143-163

Fecha de Recepción: 27-01-2020
Fecha de Aceptación: 18-04-2020
Fecha de Publicación: 05-08-2020 
OAI-PMH: http://www.indteca.com/ojs/index.php/Revista_Scientific/oai

Artículo Original / Original Article

\title{
Recreational Activities as Educational Transition Strategies
}

\begin{abstract}
The purpose of the research was to implement the recreational activity as a learning strategy that facilitates the transition process in children from initial to first grade of primary school of the basic school "María La Riva Salas", Barinas municipality, Barinas state, Venezuela. Methodologically, the investigation was framed in the socio-critical paradigm through the action research method. The informants were made up of four (4) teachers: two (2) of initial education and two (2) of primary school. The information gathering techniques were determined by the in-depth interview, with the registration of field notes. For the interpretation of information, categorization, triangulation and theorizing techniques were used. It stands out as research findings, the lack of recreational activities that allow the student to adapt to the learning situation in the first grade. Within the final reflections, there is a need to introduce changes through recreational activities in the teacher's praxis to articulate not only cognitive, bodily, motor, but also, relationships with learning, in which the student You may feel fearless, uncertainty or anxieties, facilitating their incorporation into primary education.
\end{abstract}

Keywords: educational game; learning; student adjustment.

How to cite this article:

Parra, M. (2020). Recreational Activities as Educational Transition Strategies. Revista Scientific, 5(17), 143-163, e-ISSN: 2542-2987. Recovered from: https://doi.org/10.29394/Scientific.issn.2542$\underline{2987.2020 .5 .17 .7 .143-163}$

Date Received:

27-01-2020
Date Acceptance:

18-04-2020
Date Publication:

05-08-2020 


\section{Introducción}

La educación escolar garantiza la formación integral de los niños y niñas, donde adquieren valores, actitudes, disciplina y permite la toma de decisiones propias. En el contexto de la educación inicial, la permanencia del estudiante debe cumplir con el proceso de transición que le brinde oportunidades para adaptarse al ambiente escolar de la escuela primaria; además, el mismo debe sentirse motivado para que con su asistencia adquiera aprendizajes significativos.

Desde la óptica de Valecillos (2019): "las innovaciones pedagógicas, que inundan los contextos educacionales llevan consigo elementos favorecedores de la emotividad en el individuo, para lograr que la educación, sea más humana e integral" (pág. 222). Basado en ello, el docente debe planificar actividades para llevar a la práctica estrategias innovadoras de aprendizaje que faciliten la apropiación de conocimientos y una formación integral que incluya el desarrollo de las capacidades intelectuales, motoras y afectivas del estudiante.

De esta manera, la educación se convierte a juicio de Coronel (2015a): "en el elemento esencial para la orientación de los procesos de cambio y transformación en las sociedades y lograr así un desarrollo sustentable con la formación integral y de calidad de los educando" (pág. 21). Esto, implica un proceso educativo adaptado a las necesidades de los estudiantes, tomando en cuenta el desarrollo cognitivo y el entorno como escenario para establecer interacciones diarias y alcanzar la formación integral de los mismos.

Cabe destacar, que el personal docente brinda una atención a los niños de forma afectiva para así lograr la empatía con su estudiantado, de igual forma tener en cuenta que en educación inicial los niños adquieren una variedad de conocimientos que van a ser la base de su desenvolvimiento dentro de la sociedad. Por lo tanto, se plantea la incorporación del juego como herramienta de aprendizaje en pro de favorecer la transición del niño de un 
nivel a otro, resaltando el papel del docente como mediador del proceso de enseñanza aprendizaje de los niños.

Dentro de este marco de ideas, el estudio tuvo como propósito fundamental implementar la actividad lúdica como estrategia de aprendizaje que facilite el proceso de transición en los niños y niñas de inicial a primer grado de primaria de la escuela Básica "María La Riva Salas", municipio Barinas, estado Barinas, Venezuela, para ello, se orientó bajo los postulados del paradigma sociocrítico y el método de investigación acción.

Como primer acercamiento empírico se infiere que, el docente debe planificar actividades lúdicas altamente motivadoras para llevar a cabo una adaptación que no represente la emergencia de ansiedades, incertidumbres o temores en el estudiante que recién inicia el primer grado, sino, que lo estimule a alcanzar aprendizajes significativos, mediante el juego, la socialización y la integración para lograr autonomía personal, permitiéndole, al estudiante, avanzar cognitivamente en su escolaridad.

En el contexto mundial del ámbito educativo el proceso de enseñanza aprendizaje de los niños y la niña en el nivel de educación inicial se caracteriza por generar un ambiente de calidez, confianza, amor, cooperación y respeto, donde ellos se sientan cómodos y a gusto, en la mayoría de las circunstancias el niño llega a ver a las maestras como una madre e incluso a ser un modelo a seguir en todo momento, los niños han manifestado que sus maestras tienen la razón ante sus ojos.

Respecto a las actividades lúdicas, Rodríguez (2012), citado por Posligua, Cheche y Vallejo (2017), señalan que: "el juego constituye una necesidad de gran importancia integral del niño, puesto que a través de él se adquieren conocimientos, habilidades y sobre todo, le brinda la oportunidad de conocerse así mismo, a los demás y al mundo que los rodea" (pág. 1030). Por lo tanto, el docente debe estar actualizado con estrategias activas que contribuyan a la adaptación social del estudiante a las actividades escolares. 
En tal sentido, el Sistema Educativo Venezolano debe garantizar un proceso de transición o adaptación social del estudiante desde la educación inicial para su ingreso al subsistema de educación primaria para la adquisición de destrezas como habilidades, además, de la apropiación de conocimientos que le permitan a los niños y niñas avanzar en su proceso de aprendizaje, en ese sentido, se puede iniciar mediante actividades estratégicas especiales como los juegos pedagógicos.

Conforme a ello, en los Centros de Educación Inicial, según Jiménez (2016a): "las actividades se desarrollan mediante la lúdica para que el niño se pueda expresar creativamente, participando constructivamente en las relaciones de aprendizaje" (pág. 42); además, se convierte en un sujeto activo en los diferentes espacios; mientras, en la escuela primaria, el educando se encuentra con una realidad diferente porque se cambia desde la organización del aula como la ejecución de la planificación a partir de proyectos de aprendizaje.

Por otra parte, en el ámbito educativo el proceso de enseñanza aprendizaje de los niños y las niñas, en el nivel de educación inicial, se caracteriza por generar un ambiente de calidez, confianza, amor, cooperación y respeto, donde ellos se sientan cómodos y a gusto; en la mayoría de las circunstancias, el niño llega a ver a la maestra como una madre e incluso a ser un modelo a seguir en todo momento.

No cabe duda, que el proceso de transición del preescolar al primer grado guarda relación en cuanto a la restructuración psicológica de los niños, los cuales mantienen algunas características de acuerdo a su edad de desarrollo; $\mathrm{y}$, es en este proceso continuo pero paulatino y progresivo, paralelamente Jiménez (2016b), señala que se requiere: "un tiempo de adaptación a los nuevos cambios en la vida del niño que puede durar desde los primeros meses hasta incluso todo el curso escolar, en dependencia de sus peculiaridades individuales" (pág. 3). 
Este referente resalta la importancia de considerar al niño en su proceso de transición el cual puede generarse en un tiempo prolongado, tomando en consideración sus necesidades individuales y el ambiente social donde el mismo se desarrolla. Asimismo, el Subsistema de Educación Inicial Bolivariana propuesto por el Ministerio del Poder Popular para la Educación (2007): establece la necesidad de "potenciar el desarrollo de la percepción, la memoria, la atención y la inteligencia a través de la afectividad y las diversas actividades lúdicas adecuadas a la edad" (pág. 17). Se infiere, el valor epistémico que le asigna el estado venezolano desde los entes rectores a conciertos de actividades lúdicas como estrategia del docente para desarrollar los sentidos de los niños y niñas en especial su capacidad cognitiva.

En este sentido, se debe enfatizar en la transición, la cual experimentará el niño al llegar al primer grado de educación primaria y ver un ambiente completamente nuevo para él, tanto en la distribución de los espacios de aprendizaje, como en el desarrollo de las actividades pautadas, debido a que de alguna u otra forma se destaca la formación de un ser integral en pro de la sociedad dejando a un lado la esencia e importancia de la implementación de estrategias lúdico-afectivas que fomenten el goce y disfrute del proceso de enseñanza y aprendizaje en los niveles posteriores.

En el contexto local educativo, las instituciones del municipio Barinas, no se deslinda de esta realidad, la cual se rige por las orientaciones emanadas por el Ministerio del Poder Popular para la Educación, enmarcado dentro del currículo del Subsistema de Educación Inicial y de primaria, direccionadas a las instituciones educativas por medio de la Zona Educativa (ZE), ya que este proceso de articulación y transición viene presentando fallas en todo el territorio nacional.

No obstante, en la Escuela Básica "María La Riva Salas" municipio Barinas, estado Barinas, se ha observado, en algunos niños de primer grado, la negativa a ingresar a las aulas, manifestadas por llanto y gritos, aun cuando 
la institución permite el acceso de los representantes a las instalaciones de la misma por las primeras semanas de clases. Asimismo, se observa la realización de prácticas pedagógicas apegadas a un paradigma tradicional, memorístico como repetitivo, en el cual el docente planifica actividades durante el periodo de transición de los estudiantes carentes de innovación, lúdica y participación individual o colectiva de los estudiantes, convirtiéndose en un sujeto pasivo como receptor de los saberes transmitidos por el educador.

Entre las principales causas de esta problemática planteada, se puede señalar la ausencia de estrategias que conlleven al niño al proceso de transición del nivel inicial al nivel de primaria, derivándose de ello, actitudes apáticas de los estudiantes para el desarrollo de habilidades, destrezas como conocimientos; además, dificultades para la interacción estudiante-docenteestudiante, bajo rendimiento, así como limitaciones para el establecimiento de relaciones afectivas y comunicativas eficaces, debido a la inseguridad, incertidumbre, temor o ansiedad al momento de interactuar.

Ante esta problemática, la presente investigación tiene como objetivo general: Implementar actividades lúdicas como estrategia de transición de la Educación Inicial a la Educación Primaria en los estudiantes de la Escuela Básica "María La Riva Salas", municipio Barinas, estado Barinas. Para lograr este cometido, se trazaron los siguientes objetivos específicos: Diagnosticar el nivel de conocimiento que poseen los docentes en cuanto a la aplicación de actividades lúdicas como estrategia de transición para la educación Inicial.

Asimismo, determinar el manejo de los docentes de actividades lúdicas como estrategia de transición de la Educación Inicial a la Educación Primaria. Diseñar actividades lúdicas como estrategia de transición de la Educación Inicial. De igual forma, se planteó ejecutar las actividades lúdicas como estrategia de transición de la Educación Inicial a la Educación Primaria en los estudiantes de la Escuela Básica "María La Riva Salas". Por último, se consideró evaluar la aplicación de las actividades lúdicas como estrategia de 
transición de la Educación Inicial a la Educación Primaria en los estudiantes de la Escuela Básica "María La Riva Salas, municipio Barinas, estado Barinas.

Durante el desarrollo investigativo se hizo la revisión de estudios previos, como el realizado por Sánchez (2003), citado por Hernández (2015), donde expresa la importancia de aplicar estrategias didácticas como el juego, el cual: "fortalece las destrezas y habilidades de los educandos, que en el nivel inicial ya se habían adquirido; a la vez, permite alcanzar otras para la consolidación de sus nuevos conocimientos dentro y fuera del aula" (pág. 48). Las apreciaciones del citado autor, se consideran valiosas en el contexto de la transición escolar, debido a que las actividades lúdicas le brindan oportunidades al estudiante de alcanzar una visión de continuidad en su formación integral; en consecuencia, los niños y niñas podrán reflejar una madurez en el plano cognoscitivo, social, emocional como afectivo que le permita interactuar en la escuela como con el entorno.

Desde el punto de vista teórico, la investigación se sustentó en los aportes de Naik (2014); y Park (2014), citados por Córdoba, Lara y García (2017), relacionados con las actividades lúdicas, consideradas como: "creaciones diseñadas para que los niños y niñas puedan dotar de sentido al mundo natural y social que les rodea, al mismo tiempo que se constituye en un recurso de aprendizaje y una estrategia de investigación" (pág. 83). Lo planteado por los autores, implica que los estudiantes pueden adquirir aprendizajes mediante la valorización de experiencias tomando en cuenta al otro, comprensión de la realidad, haciendo uso de la imaginación, creatividad y el juego.

Además, se tornan importantes en el aula de clase porque a través de ellas, el docente operativiza las actividades de aprendizaje para que el educando puede adquirir aprendizajes significativos, interactúan con otros pares y fortalecen aun el proceso de socialización iniciado llevado a cabo en el Centro de Educación Inicial. De allí, que las actividades de aprendizaje para 
Álvarez, González-Pienda, González-Castro y Núñez (2007), citados por Meza (2013): constituyen "guías intencionales de acción con las que se trata de poner en práctica las habilidades que establecen los objetivos del aprendizaje" (pág. 199).

Por tanto, están direccionadas hacia las habilidades desarrolladas en los estudiantes para que estos puedan apropiarse de conocimientos, los cuales deben ajustarse no solo a los objetivos a alcanzarse sino también a las necesidades, intereses, expectativas y potencialidades de los educandos, los cuales se encuentran en un período de transición escolar.

En consecuencia, el docente planificará actividades de aprendizaje sustentadas en la lúdica para la adaptación del educando a la cultura escolar como al medio intercultural, tomando en cuenta componentes afectivos mediante el desarrollo del sentido de pertenencia a la escuela, así como sentirse aceptado tanto social como académicamente, al alcanzar las expectativas de aprendizaje previamente definidas por el profesional en educación.

Tomando en cuenta estos planteamientos, se asume la definición de transición de niños de inicial a educación primaria, como lo argumenta Acevedo y Ortiz (2017a): constituyen la "[...] preparación emocional y social del niño para poder adaptarse a estos cambios, para otros, consiste en la preparación académica: dominio de conceptos básicos, lecto-escritura y matemáticas básicas, para poder enfrentar las exigencias que requiere la escolarización" (pág. 1); implicando, por consiguiente, un proceso continuo en el cual las actividades realizadas son relevantes para el estudiante, porque involucra tanto aspectos emocionales como sociales.

En este sentido, García (2010), considera que el proceso de adaptación: "es muy importante para la socialización, relación y construcción de nuevos vínculos afectivos, por tanto se requiere de un gran esfuerzo de todas las personas que entran en escena: docentes, niños y padres" (pág. 129). Para 
lograr este cometido los docentes están llamados a desarrollar habilidades afectivas y conativas que guíen el desarrollo de la personalidad del estudiante y faciliten su integración al subsistema de educación primaria.

\section{Metodología}

La orientación metodológica que direcciona el desarrollo investigativo, se sustentó en el paradigma socio-crítico, con la finalidad de conocer la realidad, de manera, se puedan introducir acciones para mejorar el entorno y buscar alternativas de solución a los problemas derivados de las prácticas educativas. De acuerdo con, Rojas (2014), en el estudio socio crítico: "el investigador y los sujetos interactúan, son activos, reflexivos y críticos" (pág. 40). Es decir, se busca dar respuestas a los problemas que se producen debido a las transformaciones sociales.

Bajo esta perspectiva, se asume como método la investigación acción con la finalidad de involucrar a los docentes en estudios, los cuales se constituyen en informantes, para transformar la realidad a partir de la reflexión de sus prácticas educativas, específicamente en las actividades lúdicas como estrategia de transición de la Educación Inicial a la Educación Primaria en los estudiantes de la Escuela Básica "María La Riva Salas" parroquia El Carmen, municipio Barinas, estado Barinas.

Se destaca, entonces, la inserción en la indagación de las fases de diagnóstico o reconocimiento de la realidad, en la cual la investigadora conoció la situación problemática a la vez que aplicó técnicas e instrumentos de recolección de información. De igual manera, se realizó una planificación, mediante el diseño de un plan de acción críticamente informado con el propósito de mejorar lo estudiado, haciendo uso de actividades lúdicas, las cuales fueron ejecutadas y evaluadas, en la medida que se llevaban a cabo, asociadas a un proceso de reflexión para la transformación del proceso de transición de educación inicial a primaria. 
Respecto a los informantes, Martínez (2006), señala que: "la muestra de informantes represente en la mejor forma posible los grupos, orientaciones o posiciones de la población estudiada, como estrategia para corregir distorsiones perceptivas y prejuicios" (pág. 137). En otras palabras, son los individuos que contribuyen a dar los elementos que integran las categorías y subcategorías.

En el presente estudio estuvo constituido por cuatro (4) docentes: dos (2) de educación inicial y dos (2) de primaria, de la institución objeto de estudio, seleccionados mediante criterios inclusivos como participación activa dentro de la institución, estudios de cuarto nivel (maestría); disposición por participar en la propuestas y actividades que se diseñaron en el plan de acción. Vale decir, que como parte de las estrategias de recolección de información se utilizó la observación, registro de notas de campo, en el caso de los informantes se les aplicó la entrevista, la cual fue realizada en un ambiente de cordialidad mediante el establecimiento de un proceso de comunicación horizontal, asertiva, asimismo, se diseñó un guion de entrevista contentiva de cinco (5) preguntas, considerando las categorías apriorísticas, como se refleja en la tabla 1.

Tabla 1. Unidad de Análisis y Categorías Apriorísticas

\begin{tabular}{|l|l|l|c|}
\hline \multicolumn{1}{|c|}{ Unidad de Análisis } & \multicolumn{1}{|c|}{ Categorías } & \multicolumn{1}{c|}{ Subcategoría } & Preguntas \\
\hline \multirow{2}{*}{ Actividades lúdicas } & \multirow{2}{*}{ 1. Tipos de juegos } & 1a.- Interacción & 1 \\
& & 1b.- Simbólicos & 2 \\
& & 1c.- De reglas & 3 \\
\hline $\begin{array}{l}\text { Transición de educación } \\
\text { inicial a primaria }\end{array}$ & \multirow{2}{*}{ 2. Preparación a la transición } & 2a.- Emocional & 4 \\
& & 2b.- Social & 5 \\
\hline
\end{tabular}

Fuente: La Autora (2018).

\section{Análisis e Interpretación de los Resultados}

Los códigos sometieron a un proceso de categorización, el cual consiste en presentar la opinión de los informantes en función de las subcategorías seleccionadas. Asimismo, se utilizó la triangulación de la información para 
presentar la postura de la investigadora sobre las subcategorías emergentes. Finalmente, se estableció la teorización de los hallazgos en contraste con las teorías consultadas, parte del poroso es presentado en la tabla 2.

Tabla 2. Opinión emitida por los docentes tomando en cuenta las categorías apriorísticas.

\begin{tabular}{|c|c|c|c|c|}
\hline $\begin{array}{l}\text { Unidad de } \\
\text { Análisis }\end{array}$ & Categoría & Pregunta & Docente Inicial & $\begin{array}{l}\text { Docente } \\
\text { Primaria }\end{array}$ \\
\hline $\begin{array}{l}\text { Actividades } \\
\text { Lúdicas }\end{array}$ & $\begin{array}{c}\text { Tipos de } \\
\text { juego }\end{array}$ & $\begin{array}{l}\text { ¿Qué tipo de } \\
\text { actividades } \\
\text { introduce para } \\
\text { lograr la } \\
\text { interacción del } \\
\text { niño con el } \\
\text { medio que le } \\
\text { rodea? }\end{array}$ & $\begin{array}{l}\text { D1: Actividades } \\
\text { lúdicas, sobre todo } \\
\text { en el espacio de } \\
\text { experimentar y } \\
\text { descubrir } \\
\text { D2: Diferentes tipos } \\
\text { de actividades como } \\
\text { dramatizaciones, } \\
\text { diálogos, resolución } \\
\text { de problemas. }\end{array}$ & $\begin{array}{l}\text { D3: Solo las } \\
\text { relacionadas } \\
\text { con el proyecto } \\
\text { de desarrollo } \\
\text { endógeno } \\
\text { D4: Las } \\
\text { actividades que } \\
\text { planifico se } \\
\text { relaciona con el } \\
\text { huerto escolar }\end{array}$ \\
\hline $\begin{array}{l}\text { Transición de } \\
\text { educación } \\
\text { inicial a } \\
\text { primaria }\end{array}$ & $\begin{array}{c}\text { Preparación } \\
\text { a la } \\
\text { transición }\end{array}$ & $\begin{array}{l}\text { ¿Cuáles son tus } \\
\text { expectativas } \\
\text { sobre la } \\
\text { preparación } \\
\text { social del niño } \\
\text { hacia su ingreso } \\
\text { a educación } \\
\text { primaria? }\end{array}$ & $\begin{array}{c}\text { D1: Se continúe } \\
\text { afectiva y } \\
\text { pedagógicamente el } \\
\text { aprendizaje en los } \\
\text { niños } \\
\text { D2: La obtención de } \\
\text { aprendizajes } \\
\text { significativos }\end{array}$ & $\begin{array}{c}\text { D3: Se } \\
\text { familiarice con } \\
\text { la educación } \\
\text { primaria } \\
\text { D4: Desarrollar } \\
\text { habilidades y } \\
\text { destrezas para } \\
\text { la vida } \\
\end{array}$ \\
\hline
\end{tabular}

Fuente: La Autora (2018).

En las opiniones emitidas por los docentes, se revela lo siguiente: En relación a la Unidad de Análisis Actividades Lúdicas, en la categoría tipos de juego, específicamente a los juegos de interacción, se evidencia los docentes de educación inicial señalan el empleo de actividades lúdicas en diferentes espacios de aprendizaje, como es el espacio de experimentar y descubrir; además, indican que hacen uso de dramatizaciones, resolución de problemas, diálogos, experimentos, entre otros. Mientras, las profesionales en educación primaria, lo circunscriben a prácticas educativas relacionadas con los proyectos de desarrollo endógeno, específicamente, en el trabajo con el huerto escolar. 
Al Respecto, Coronel (2015b), argumenta que: "el juego proporciona nuevas formas de explorar la realidad y estrategias diferentes para trabajar sobre la misma" (pág. 34); además, permite que el estudiante pueda interactuar con el entorno, pares y demás miembros de la escuela-familiacomunidad, siendo fuente de aprendizaje al establecer relaciones con el medio social en el cual se desenvuelve el educando. Asimismo, el estudiante puede practicar lo aprendido y generar discusiones socializadas sobre las actividades realizadas.

En relación con la Unidad de Análisis-Transición de educación inicial a primaria, en la categoría proceso de adaptación, las expectativas son muchas en los docentes, especialmente porque se le estaría dando respuesta a las demandas de los estudiantes y estos no se sentirían frustrados, familiarizados con el grado que cursa, además, se identifica con la escuela.

En este mismo orden de ideas, Acevedo y Ortiz (2017b), aportan consideraciones educativas sobre el proceso de transición, indicando que dentro de los factores que intervienen es significativo: "involucrar no sólo al niño sino a quienes lo acompañan en este proceso, los padres de familia. Se refiere también a los esfuerzos constantes y continuos que la instancia educativa hace para vincular el ambiente natural del niño" (pág. 12). Vale decir, que los niños y niñas son los protagonistas de este proceso. De igual forma, se infiere el interés de los docentes por generar acciones estratégicas para incorporar a los padres y representantes de forma activa a las actividades de aula mediante el dialogo asertivo.

\subsection{Planificación, Ejecución y Evaluación de las actividades lúdicas} como estrategia de transición de la Educación Inicial a la Educación Primaria

Las actividades planificadas se diseñaron a partir del diagnóstico aplicado a los docentes con el propósito de generar escenarios de reflexión 
sobre la praxis educativa llevada a cabo y transformar la misma, como se muestra a continuación en la tabla 3.

Tabla 3. Plan de acción, actividad lúdica. Sensibilización a los docentes.

Finalidad: Sensibilizar mediante un círculo de formación docente sobre la importancia de las actividades lúdicas como estrategia de transición de educación inicial a educación primaria

\begin{tabular}{|c|c|c|c|c|}
\hline \multicolumn{2}{|c|}{$\begin{array}{l}\text { Contenido } \\
\text { - Actividades lúdicas } \\
\text { - Transición de } \\
\text { educación inicial a } \\
\text { primaria } \\
\text { Estrategia: } \\
\text { - Circulo de Formación }\end{array}$} & \multicolumn{3}{|c|}{$\begin{array}{l}\text { Actividades } \\
\text { - Saludo de bienvenida por parte de la investigadora } \\
\text { - Realización de dinámica "Pedro llama a Pablo" } \\
\text { Objetivo de la dinámica: Recordar los nombres de los } \\
\text { participantes. } \\
\text { Orientaciones: } \\
\text { 1. Conformación de equipos de trabajo. } \\
\text { 2. Conceptualizaciones de actividades lúdicas, Importancia de } \\
\text { actividades lúdicas para la transición de etapas. } \\
\text { 3. Compendio de actividades lúdicas. } \\
\text { 4. Ejecución y evaluación de las actividades lúdicas } \\
\text { seleccionadas por los equipos de trabajo. } \\
\text { Realización y lectura de conclusiones por equipos de trabajo } \\
\text { - Plenaria para la lectura de conclusiones. } \\
\text { - Realimentación y evaluación de la actividad. }\end{array}$} \\
\hline & & & & nació \\
\hline & & & 19/10/2018 & $\begin{array}{l}\text { Asistencia. } \\
\text { Evaluación de rasgos participativos } \\
\text { individuales y colectivos. } \\
\text { Aportes significativos al conocimiento. } \\
\end{array}$ \\
\hline
\end{tabular}

Fuente: La Autora (2018).

\subsection{Ejecución y evaluación de la propuesta}

Con el compromiso de participación activa coherente y afectiva del personal docente que labora en Escuela Básica "María La Riva Salas" municipio Barinas, estado Barinas, se procedió a informar sobre los objetivos propuestos en la investigación, seguidamente, se describió el plan de acción diseñado invitando a realizar las actividades bajo un ambiente cordial sin temores y limitaciones, para ello, se utilizó como estrategia la conformación de círculos para la presentación y discusión de cada objetivo de la propuesta desarrollando las actividades en función de las orientaciones pedagógicas de la investigadora. 
Una vez cumplidas las dinámicas de juegos lúdicos, se les pregunto a los participantes ¿Les gustó?, respondiendo una docente asistente: Sí, porque es una forma de introducir la motivación a las actividades formativas, haciendo uso del juego. Inmediatamente, la investigadora procedió a dar inicio al círculo de formación, haciendo lectura y análisis de las diferentes conceptualizaciones de actividades lúdicas y sobre la importancia de las mismas para el proceso de transición de los estudiantes; asimismo, señaló que la transición experimentada por el niño que egresa de educación inicial hacia la primaria, constituye un proceso de cambio para favorecer la adaptación social, académica y emocional del estudiante a la escuela, con la finalidad que éste alcance aprendizajes significativos.

Para ello, el docente debe planificar y ejecutar actividades que coadyuven a la adaptación sin causar, temores, ansiedades o incertidumbre a los estudiantes, de manera, pueda alcanzar mayores niveles de maduración social, desarrollo intelectual haciendo uso de actividades lúdicas; ofreciendo un ambiente que le permita manipular diferentes recursos, además, el aula este organizada para favorecer la interacción.

En referencia a la valoración de las actividades cumplidas, se apreció un espíritu participativo, interés por perfeccionar las dinámicas de juego, claridad para comunicar las experiencias del aula, asimismo, se evidencio la aplicación de conocimiento pedagógico para construir un modelo cognitivo, conativo y afectivo que optimice la transición escolar del nivel inicial. Por otra parte, y a solicitud de los docentes, se planificaron actividades para ser llevadas a cabo en el aula de clases, como se evidencia en la tabla 4.

Tabla 4. Actividad Lúdica: Juego de Roles.

\begin{tabular}{|l|l|}
\hline $\begin{array}{l}\text { Finalidad: Facilitar a la docente el juego de roles para la identificación conscientemente de } \\
\text { reglas, obligaciones y deberes a cumplirse en la escuela. }\end{array}$ \\
\hline \multicolumn{1}{|c|}{ Contenido } & \multicolumn{1}{|c|}{ Actividades } \\
- Reglas & - Saludo de bienvenida y presentación de la \\
- Obligaciones & investigadora los estudiantes. \\
- Deberes a cumplir en la escuela & - Realización de dinámica rompe hielo: "Los \\
\hline
\end{tabular}


OAI-PMH: http://www.indteca.com/ojs/index.php/Revista_Scientific/oai

Artículo Original / Original Article

- Juego de roles

sombreros".

- Orientaciones sobre el juego de roles a realizarse.

- Realización de la actividad mediante el juego de roles.

- Realimentación y evaluación de la actividad.

\begin{tabular}{|c|c|c|l|}
\hline Responsable & Duración & Fecha & \multicolumn{1}{c|}{ Evaluación } \\
\hline Investigadora & $\begin{array}{c}180 \\
\text { minutos }\end{array}$ & $23 / 10 / 2018$ & $\begin{array}{l}\text { Instrumento: Registro descriptivo. } \\
\text { Técnica: Observación directa. } \\
\text { Indicadores: Participación, actitud ante el } \\
\text { cambio, motivación, interés. }\end{array}$ \\
\hline
\end{tabular}

Fuente: La Autora (2018).

Esta actividad se realizó en las instalaciones de la Escuela Básica "María La Riva Salas", parroquia El Carmen, municipio Barinas, estado Barinas, específicamente en el primer grado, sección "A", el día martes 23 de octubre del año 2018, en el turno de la mañana, con la finalidad de facilitar a la docente el juego de roles para la identificación conscientemente de reglas, obligaciones y deberes a cumplirse en la escuela.

Al finalizar esta dinámica, la docente preguntó a los estudiantes ¿Cómo se sintieron?, una estudiante señaló: como en preescolar, allí nos divertíamos y aprendíamos; seguidamente otro estudiante manifestó: Me gustó, porque no me senté en esa silla que no me gusta. Inmediatamente, la profesional en educación explicó la existencia en la institución de unos acuerdos de convivencia que cada miembro de la escuela debe acatar.

En este sentido, la convivencia viene dada por las interacciones que se establecen tanto en el aula como en la escuela, familia y comunidad, basadas en el diálogo, respeto mutuo, valores, entre otros. Culminado el juego de roles, los estudiantes mostraron satisfacción por la actividad realizada, señalando algunas reglas para cumplirlas durante el desarrollo de las actividades de aula, mediante un intercambio de ideas. Se destaca, la existencia de participación, motivación por parte de los estudiantes; además, la docente del primer grado se mostró receptiva ante la alternativa para la transición de educación inicial a primaria. 


\section{Reflexiones finales}

El estudio permitió diagnosticar la situación problemática en la Escuela Básica "María La Riva Salas", donde se evidencio la actitud clave de los docentes por generar en el aula acciones estratégicas como el uso de dramatizaciones para fortalecer los niveles cognitivos de los estudiantes, así como, el desarrollo de diálogos y experimentos simples. En referencia a la planificación y ejecución, se logró realizar actividades para la transformación de la praxis educativa con la participación de docentes de educación inicial, primer grado y estudiantes de primer grado, demostrando que las actividades lúdicas sirven como estrategia para alcanzar la adaptación de los estudiantes en su nueva etapa escolar.

Resulta positiva la incorporación de juegos pedagógicos en la elaboración de las estrategias orientadoras y su vinculación con el aprendizaje del niño y niña facilita su activación cognitiva. Por tanto, los contenidos referentes a los juegos recreativos y los trabajos manuales constituyen una vía de motivación tanto para los estudiantes como para los padres y representantes, ya que en los eventos grupales de trabajo se observa un nuevo estado de ánimo que permite el desenvolvimiento total de los niños y niñas en las metas que se desean lograr en las tareas.

Mediante la ejecución del plan de acción se proporcionó a los docentes objeto de estudio, los diálogos formativos y estrategias en función de los valores educativos, observando en los participantes reflexión, interés, responsabilidad en el proceso de enseñanza y aprendizaje de los estudiantes, demostrado en los días en curso en el aula de clase, donde las manifestaciones se dieron de forma positiva para cada uno de los niñas y niñas; mostrando motivación y avance en su actitud coherente con su nivel cognitivo y afectivo.

Considerando lo planteado, se sugiere a la institución educativa la planificación de círculos de formación direccionados hacia estrategias de 
aprendizaje para alcanzar la adaptación del educando a este nivel educativo. Asimismo, se recomienda a otros investigadores profundizar la problemática estudiada mediante el desarrollo de basamentos teóricos, axiológicos y ontológicos de las actividades lúdicas como estrategias de transformación cognitiva, afectiva y educativa en el contexto de educación inicial.

\section{Referencias}

Acevedo, B., \& Ortiz, F. (2017a,b). Proceso de transición de los niños y niñas del III nivel de Educación Inicial al Primer Grado del Colegio Público Poder Ciudadano Benjamín Zeledón Rodríguez, ubicado en el departamento de Managua, distrito III, durante el II Semestre 2016. Tesis. Managua, Nicaragua: Universidad Nacional Autónoma de Nicaragua, Recinto Universitario "Rubén Darío" Recuperado de: http://repositorio.unan.edu.ni/3808/1/51788.pdf

Coronel, D. (2015a,b). El juego lúdico como estrategia didáctica para la enseñanza de la lectura en los niños y las niñas de primer grado. Tesis de Maestría. Naguanagua, Venezuela: Facultad de Ciencias de la Educación de la Universidad de Carabobo. Recuperado de: http://mriuc.bc.uc.edu.ve/bitstream/handle/123456789/1348/coronel.pd f? sequence $=1$

Córdoba, E., Lara, F., \& García, A. (2017). El juego como estrategia lúdica para la educación inclusiva del buen vivir. ENSAYOS. Revista de la Facultad de Educación de Albacete, 32(1), 81-92, e-ISSN: 2171-9098. Recuperado de:

https://revista.uclm.es/index.php/ensayos/article/view/1346

García, I. (2010). Procesos de adaptación de los niños de temprana edad en la Institución Educativa Mañanitas. Trabajo de grado. Caldas, Colombia: Corporación Universitaria Lasallista. Recuperado de: http://repository.lasallista.edu.co/dspace/bitstream/10567/652/1/Proces 
os adaptacion.pdf

Hernández, M. (2015). Estrategias didácticas empleadas por los docentes en la transición escolar entre los niveles de educación inicial y primaria del NER 295, municipio Arístides Bastidas, estado Yaracuy. Revista Arbitrada del CIEG, (20), 45-61, e-ISSN: 2244-8330. Recuperado de:

http://www.grupocieg.org/archivos revista/Ed.\%2020\%20(45-

61)\%20Hern\%C3\%A1ndez\%20Ortiz\%20\%20Junio\%202015 articulo id174.pdf

Jiménez, C. (2016a,b). Pedagogía de la creatividad y de la lúdica. Bogotá, Colombia: Editorial Magisterio.

Martínez, M. (2006). La investigación cualitativa (Síntesis Conceptual). Revista IIPSI, 9(1), 123-146, e-ISSN: 1560-909X. Recuperado de: http://sisbib.unmsm.edu.pe/bvrevistas/investigacion psicologia/v09 n1 /pdf/a09v9n1.pdf

Meza, A. (2013). Estrategias de aprendizaje. Definiciones, clasificaciones e instrumentos de medición. Propósitos y Representaciones, 1(2), 193-213, ISSN: 2307-7999; e-ISSN: 2310-4635. Recuperado de:

http://dx.doi.org/10.20511/pyr2013.v1n2.48

Ministerio del Poder Popular para la Educación (2007). Subsistema de Educación Inicial Bolivariana: Currículo y Orientaciones Metodológicas. ISBN: 978-980-218-282-4. Caracas, Venezuela: Fundación Centro Nacional para el Mejoramiento de la Enseñanza de Ciencia, CENAMEC.

Posligua, J., Cheche, W., \& Vallejo, B. (2017). Incidencia de las actividades lúdicas en el desarrollo del pensamiento creativo en estudiantes de educación general básica. Dominio de las Ciencias, 3(3), 10201052, e-ISSN: 2477-8818. Recuperado de: 
Rojas, B. (2014). Investigación cualitativa, fundamentos y praxis. ISBN: 980-273-471-3. Caracas, Venezuela: Fondo Editorial de la Universidad Pedagógica Experimental Libertador - FEDUPEL.

Valecillos, B. (2019). Desde la pedagogía de la ternura: inicio de lo lógicomatemático en preescolar. Revista Scientific, 4(12), 220-239, e- ISSN: 2542-2987. Recuperado de:

https://doi.org/10.29394/Scientific.issn.2542-2987.2019.4.12.11.220$\underline{239}$ 


\section{Artículo Original / Original Article}

\section{Marlise del Valle Parra Peña}

e-mial: mdvpp4@gmail.com

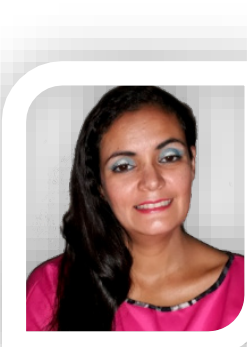

Nacida en Barinas, Venezuela, el 4 de julio del año 1983.

Egresada como TSU en Educación Inicial del Instituto Universitario de Tecnología "Coronel Agustín Codazzi" (IUTAC, 2000); posteriormente, me desempeño como Profesor de Educación Inicial de la Universidad Pedagógica Experimental Libertador (UPEL-IMPM, 2010), extensión Académica Barinas, estado Barinas; además, laboro como docente de aula en el Centro de Educación Inicial Bolivariano María La Riva Salas. 